University of San Diego

Digital USD

Spring 5-26-2018

\title{
Prevention of Functional Decline in Hospitalized Geriatric Patients: An Evidence-based Mobility Pilot Project on an Acute Care Elderly (ACE) Unit
}

Hannah Grushkowsky

University of San Diego, hannahsa@sandiego.edu

Follow this and additional works at: https://digital.sandiego.edu/dnp

Part of the Nursing Commons

\section{Digital USD Citation}

Grushkowsky, Hannah, "Prevention of Functional Decline in Hospitalized Geriatric Patients: An Evidencebased Mobility Pilot Project on an Acute Care Elderly (ACE) Unit" (2018). Doctor of Nursing Practice Final Manuscripts. 69.

https://digital.sandiego.edu/dnp/69

This Doctor of Nursing Practice Final Manuscript is brought to you for free and open access by the Theses and Dissertations at Digital USD. It has been accepted for inclusion in Doctor of Nursing Practice Final Manuscripts by an authorized administrator of Digital USD. For more information, please contact digital@sandiego.edu. 
Prevention of Functional Decline in Hospitalized Geriatric Patients:

An Evidence-based Mobility Pilot Project on an Acute Care Elderly (ACE) Unit

Hannah S. Grushkowsky, MSN, RN, PHN, CNL

University of San Diego

Hahn School of Nursing 
Prevention of Functional Decline in Hospitalized Geriatric Patients: An Evidence-based Mobility Pilot Project on an Acute Care Elderly (ACE) Unit

\section{Introduction}

\section{Clinical Problem}

Functional decline, defined as the ability to perform activities of daily living (ADL), is the leading complication of hospitalization in the elderly (Ahmed \& Pearce, 2010; Creditor, 1993; Graf, 2006; Kleinpell, Fletcher, \& Jennings, 2008; Kosse, Dutmer, Dasenbrock, Bauer, \& Lamoth, 2013; Krall et al., 2012; Padula, Hughes, \& Baumhover, 2009; Valiani et al., 2016). Hospitalized older adults spend only $3 \%$ of the time walking or standing (Hastings, Sloane, Morey, Pavon \& Hoenig, 2014). Loss of functional independence results from the inability to maintain function during hospitalization compounded by acute illness (Kleinpell et al., 2008). It is estimated that $23-33 \%$ of hospitalized older adults experience decreased mobility. From a study of 15,498 hospital records, one out five ambulatory patients on admission became impaired during hospitalization (Valiani et al., 2016). One third of adults 70 and older are discharged with a major new disability not present on admission. A follow-up one year later demonstrated that less than $50 \%$ of older adults with a hospital associated disability recover to their original level of function (Hastings et al., 2014).

Complications of bedrest affect all body systems. Cardiovascular problems include orthostatic hypotension, thromboembolism, increased workload of the heart, and a plasma volume decrease of 500 milliliters. Respiratory problems include atelectasis, stasis of secretions, and oxygen-carbon dioxide imbalance. Musculoskeletal problems include loss of muscle mass, muscle strength decrease of $5 \%$ of muscle mass a day, bone loss, and contractures. Gastrointestinal and genitourinary complications include constipation, incontinence, 
malnutrition, and dehydration. Skin problems include pressure ulcers. Lastly, psychological problems include depression, disorientation, and delirium (Ahmed \& Pearce, 2010; Creditor, 1993; Graf, 2006; Hastings et al., 2014; Kleinpell et al., 2008; Kosse et al., 2013; Olson,

Johnson, \& Thompson, 1990; Wood et al., 2014). The negative effects of bedrest that encompass physical, mental, and psychological decline often result in negative patient outcomes such as adverse hospital events like increased length of stay (LOS), readmissions, morbidity, and added discharges to skilled nursing facilities (SNFs) (Ahmed \& Pearce, 2010; Creditor, 1993; Hastings et al., 2014; Kleinpell et al., 2008; Krall et al., 2012; Padula, et al., 2009; Valiani et al., 2016; Wood et al., 2014).

\section{Project and Context}

Geriatric patients 65 and older in an acute care hospital on a medical surgical unit did not have a mobility protocol. Anticipated barriers noted in the literature included users excluding patients because the patients may be perceived to be cognitively and physically impaired. Some nursing staff assist older adults with activities of daily living (ADL) simply to save time, even when the patients are capable of performing them independently (Wallace \& Shelkey, 2008). Therefore, it is critical that all nursing staff encourage older adults to remain as independent as possible. Other potential barriers include lack of motivation, high patient acuities, low staffing ratios, time constraints, and the use of paper evidence-based practice (EBP) tool charting. The plan to overcome some of these challenges included sharing positive patient outcomes from EBP, staff buy-in prior to the implementation of the project, and reassessment of the project every week during auditing. Facilitators included a Geriatric Rounding Team twice a week, an established Acute Care Elderly (ACE) unit that focuses on the needs of geriatric patients, 
Geriatric Resource Nurses (GRNs) dedicated to the needs of the elderly, a highly motivated staff, and a Department Administrator who is Head of Mobility in the hospital.

\section{Methods}

\section{Research Synthesis}

The review of literature was conducted through using electronic database sources including PubMed and CINHAL. Keywords in database searches included "mobility," "older adult," "activities of daily living," "Katz, "hospitalization," "acute care," "functional decline," “elderly," and "geriatric." In PubMed the studies were limited to humans. Different searches were done that yielded about 150 articles each time. A total of 66 articles were reviewed. Eight systematic reviews, six randomized control trials, eight pilot studies, four qualitative studies, 20 cohort and observational studies, eight overview articles on functional decline in the elderly, and seven articles on EBP mobility tools were used. The following pilot studies that will be reviewed are considered Level IV evidence. The research evidence supports nursing staff education tailored for geriatric patients, inclusion of the family in the care of the patient, and encouraging mobilization through walking programs to prevent functional decline during hospitalization (Kleinpell et al., 2008).

\section{Iowa EBP Model}

The Iowa model was chosen because it guides clinicians in making decisions that affect patient outcomes through using EBP in inpatient settings (Titler, 2010). The model includes a flowchart for decision making through problem-based steps and feedback loops to guide change (Schaffer, Sandau, \& Diedrick, 2012). Assumptions of the model include working as a group, performing an evaluation, and several steps to align clinicians' behavior and system support to provide EBP healthcare (Titler, 2010). Intended users include practitioners and interdisciplinary 
teams (Titler, 2010). Continuous quality improvement tools used included a driver diagram, PDSA model, and control charts for outcomes data. Strategic planning included assessment, setting direction, refining an adoption plan, implementation, and evaluation. A quick check assessment included mission and purpose, need and feasibility of project, and a SWOT analysis (Sare \& Ogilvie, 2010). For the Iowa Model, the problem and knowledge triggers that were used included risk management data, identification of a clinical problem, and new research. The topic was considered a priority for the organization.

\section{Supporting EBP}

EBP data includes several pilot studies. The first study was a three-month quality improvement early mobility program pilot $(n=521)$ that was implemented on 32-bed acute care medical unit by a seven-member interdisciplinary team from April to October 2012. Results included decreased readmission and fall rate and the same number of pressure ulcers. The study did report an increased LOS that may have been due to the severity of the case mix (Wood et al., 2014). The second study, STRIDE (assisted early immobility for hospitalized older veterans) $(n=92)$ supervised a 20 -minute walking program for veterans compared to usual care $(n=35)$ from February to July 2012. Post-intervention outcomes included no difference in readmission rates, one fall in each group, decreased in LOS (STRIDE 4.7 days vs. usual care 5.7 days), and more patients discharged home (STRIDE 92\% vs. usual care 76\%) (Hastings et al., 2014). The third study consisted of a transdisciplinary team led by a Clinical Nurse Specialist with the intervention to assist in ADLs. Part of the intervention included walking the patient two times a day and placing the patient in a chair for all meals. ACE patients $(n=90)$ were compared to usual care $(n=395)$ over three months. Results showed no falls, pressure ulcers, urinary tract infections, minimal restraint use, decreased LOS (3.18 vs.3.90), improved function level (Katz average 
score on admission $=1.73$ vs. discharge $=2.67$ ), and increased patient, family, and staff satisfaction (Krall et al., 2012). The fourth study had 25 patients in the intervention and 25 in the the control. The certified nursing assistant (CNA) was given the priority to walk the patients two to four times a day, place the patient to the chair for meals, and assist in toileting. Barthel was the EBP tool used for self-perceived functional level at time of admission, two weeks prior to admission, and at discharge. The Barthel preadmission scores (perception) ranked higher on admission for both groups. Discharge scores improved from admission baseline for the intervention compared control group. The control group had a significant decrease in function between preadmission to discharge ( $p=.006)$. An Up and Go Test was given to both groups. The scores improved slightly in both groups for admission and discharge, but was not statistically significant. Greater improvement in function $(p=0.05)$ and reduced LOS $(p<.001)$ were noted in the intervention compared to control. In addition, ambulating in hall was found to be more effective than chair rest and ambulating in the room (Padula, et al., 2009).

\section{Evidence-based Intervention}

A mobility-based protocol created by a Doctor of Nursing Practice (DNP) student with interdisciplinary team input was implemented for three months on a 8 to12-bed geriatric medical-surgical ACE unit from September 12, 2016 to December 15, 2016. The research literature supports EBP outcomes of ACE units in decreasing LOS, functional decline, discharges to SNFs, and delirium (Kleinpell et al., 2008; Krall et al., 2012). ACE goals include preventing complications of hospitalization, promoting maintenance of functional level, promoting mobility, and implementing strategies to discourage use of restrictive devices (Krall et al., 2012). The EBP tool, Katz Index of Daily Living Index (Katz tool), measures functional status of patient's ability to perform ADLs independently. There are six functions: bathing, 
dressing, toileting, transferring, continence, feeding. A score of 1 is independent with no supervision, direction, or personal assistance. A score of 0 is dependent with supervision, direction, personal assistance, or complete care (Katz, Downs, Cash, \& Grotz, 1970; Krall et al., 2012).

The inclusion criteria were ambulatory patients who were age 65 and older on admission. Exclusion criteria were adults less than 65 years old, non-ambulatory patients, orders for bedrest or restricted mobility, hemodynamic instability, patients admitted from SNFs, alcohol or drug withdrawal, patients who were suicidal, refusal by the patient or family, and orders for hospice, palliative, or comfort care. The protocol was implemented by the registered nurses (RNs) and nursing assistants (CNAs) that would function as mobility aides. They mobility protocol was to ambulate the patient at minimum twice a day, place the patient in a chair for all meals, educate the patient and family about the hazards of immobility through an educational handout created by the DNP student, and monitor their ADLs on admission and discharge through the EBP Katz tool. 


\section{Project Planning}

\section{Figure 1 Driver Diagram Mobility Protocol on an Acute Care Elderly Unit}

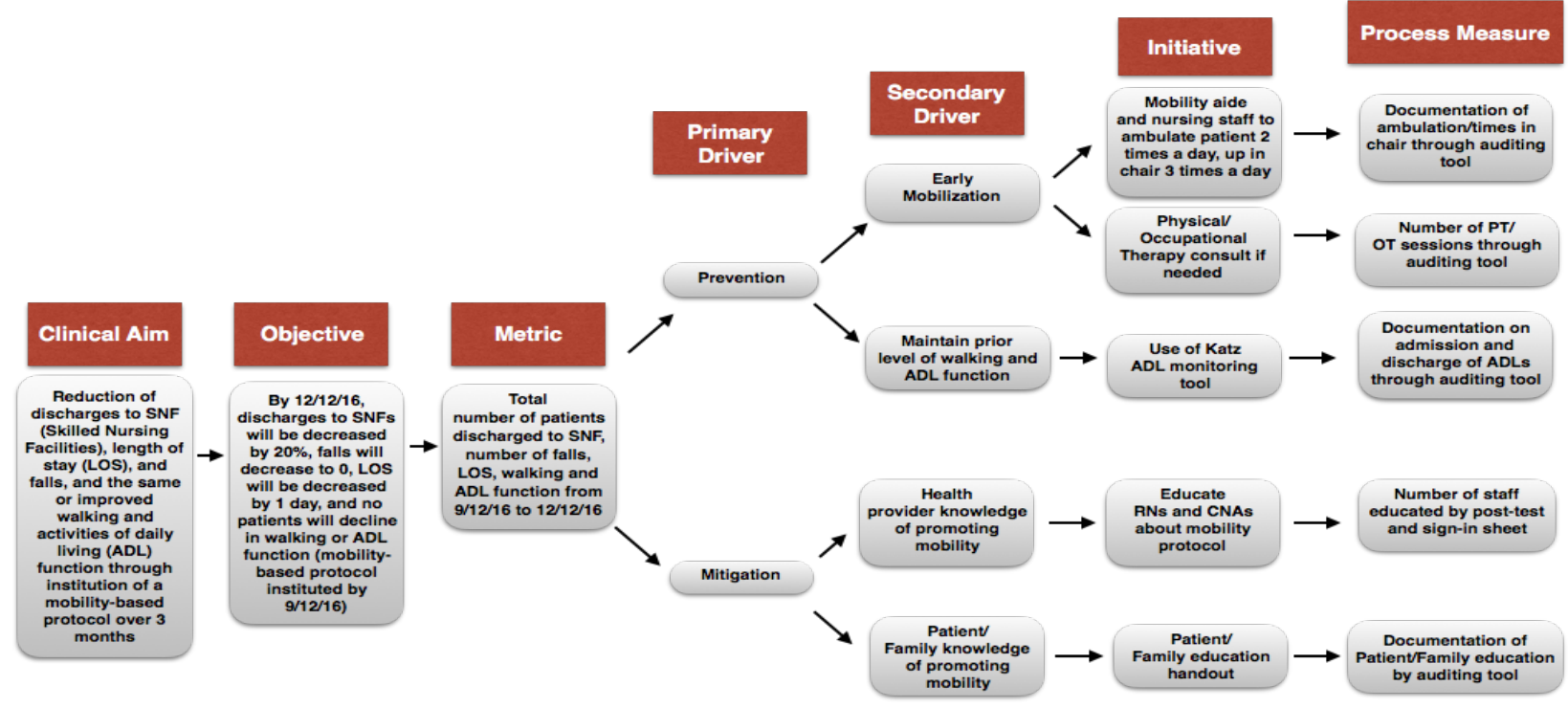

Sufficient research and evidence was collected by the DNP student and presented to the student Advisor Dr. Fry-Bowers, Data Analyst, Dr. Dee-Canon, and the Geriatric Clinical Nurse Specialist (GCNS). The project was reviewed by the Institutional Review Boards of the University of San Diego and Kaiser Permanente Southern California with the determination that the project was not human research and was granted exemption. Prior to the implementation of the project, interdisciplinary input was sought from the Assistant Department Administrator and Nurse Educator, the Department Administrator, Geriatric Resource Nurses (GRNs), the Department Based Team (DBT), Head of Physical Therapy, Patient Education Committee, and Patient Member Committee. One month prior to the project initiation, a reminder was placed on every computer with a go live date detailing inclusion, exclusion, and the mobility protocol to alert the staff that the mobility project was approaching. In addition, the reminder on the computer served as a reference for screening criteria during the admission of patients. One week of baseline data was collected prior to staff education. Ten patients were identified by the DNP 
student that would have qualified for the study from September 9-September 16. Data that was collected included current living situation, discharge destination, time and date of admission and discharge, number of times in chair, number of walks in 24 hours, distance of walk, falls, and physical therapy (PT) or Occupational Therapy (OT) referral.

The EBP mobility protocol was designed based on previous EBP studies. One-on-one staff education on the mobility protocol was conducted by the DNP student or GCNS to 17 registered nurses (RNs) and 10 certified nursing assistants (CNAs). The RNs were educated on the background of mobility in geriatric patients, supporting evidence, complications of immobility, project goals, inclusion criteria, intervention, documentation, barriers, EBP tool, and education time served as an opportunity to ask questions. A written post-test was given regarding the information with case scenarios and an individual review of the answers. CNAs were individually educated on who qualifies for the study, the intervention, and how to assist geriatric patients with ADLs with a verbal questionnaire given at the end of education. Education was done during working hours and all education completed prior to go live date.

\section{Data Collection Procedure}

Data that was collected included age, sex, diagnosis, current living situation, discharge destination, time and date of admission and discharge, number of times in chair, number of walks in 24 hours, distance of walked, falls, PT or OT referral, and Katz preadmission, admission, and discharge score. The process was evaluated weekly and monthly for the first two months by the DNP student. The last month of data was analyzed a month later. Month 1 data was reviewed one-on-one with staff and they had the opportunity to ask questions and make recommendations. Month 2 data was placed in the unit huddle book and signatures were required by the staff to ensure the information was read. Month 2 data was also presented in the DBT. 
On admission, the RN would screen each patient and a walking man magnet in purple was placed next to the patient's name on the admission board to notify the staff that the patient was included in the study. If the RN was not regular staff, the charge $\mathrm{RN}$ would screen the patient. The RN was responsible for charting the current level of function on admission, every shift, and discharge. The RN was to chart ambulation, feet walked, assistive device used, when the patient was in the chair, and PT session ambulation. The care plan for mobility was added to the chart by the RN and an education handout on the hazards of immobility created by the DNP student was given to the patient and the family and was discussed and documented in the electronic medical record. The Katz tool was completed by the $\mathrm{RN}$ regarding ADL function prior to admission, during admission, and on discharge. The admission portion was observed by the RN or ADL function was reported by the CNA to the RN in 24 hours and documented on the Katz tool. The Katz tool was a paper flyer that was placed on the patient's careboard on a specialized clip so it would not be lost. It was collected on discharge by the discharge $\mathrm{RN}$ and placed in the mobility folder for review by the DNP student. The mobility folder was available on the unit as a reference for the staff on education discussed, Katz tool copies, and copies of the educational handout.

\section{Results}

There were 66 patients that met initial inclusion criteria. Thirteen patients were excluded. Six patient refused three times to walk or be in a chair, one patient was incorrectly identified for the study and was not ambulatory, two patients changed criteria to palliative care or hospice, and four patients were transferred to other units. There were a total of patients 53 patients. Overall results demonstrated no discharges to SNFs, no patients experienced a fall, length of stay was decreased, ADL function was maintained or improved, and ambulatory function was maintained or improved. During the study, $84 \%$ of patients and family were educated. 


\section{Figure 2}

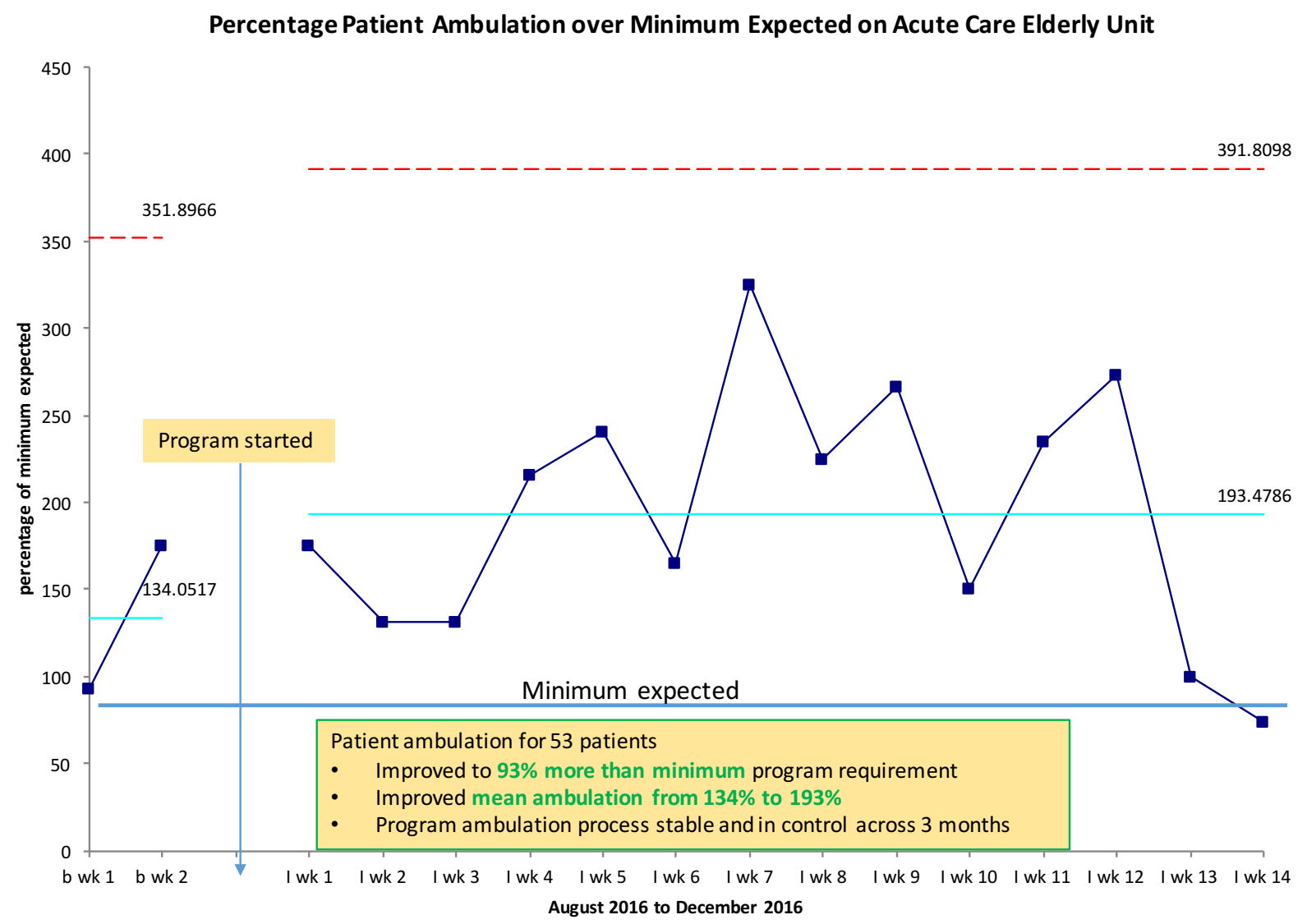

Mean ambulation prior to the intervention was $100 \%$, which demonstrated that the patients were already being ambulated. The control chart shows that clearly over course of study, the unit had made it apart of customary practice for 14 weeks to ambulate the patient more than twice. With the intervention, mean ambulation was $93 \%$ more than minimum and mean ambulation range was $134 \%$ to $193 \%$, with a maximum high point of about $325 \%$. This change was sustained by the staff even when the DNP student was not present during December. 


\section{Figure 3}

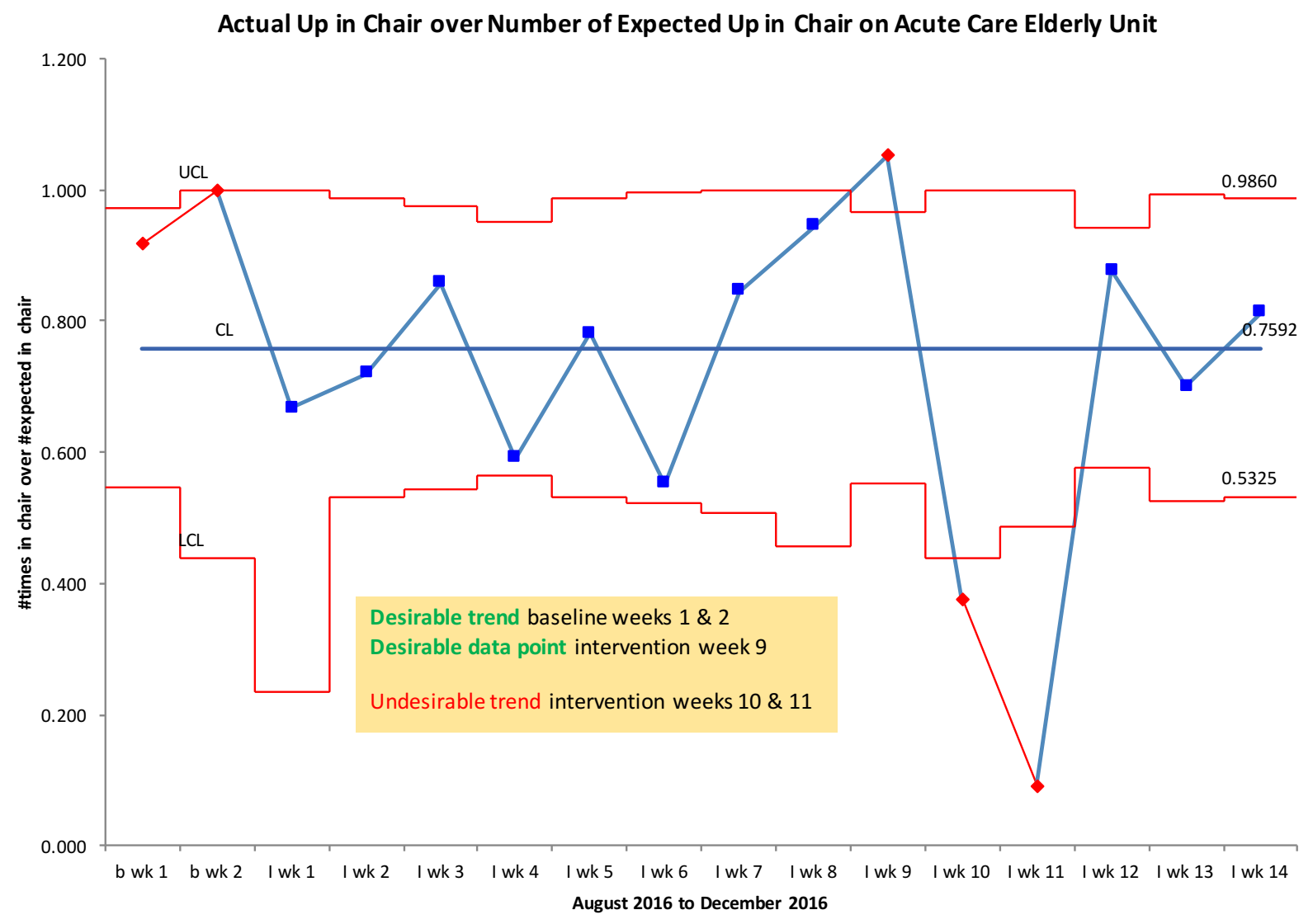

It was expected that the patients would be in the chair three times a day. Prior to the intervention, patients were mostly in the chair during baseline week 1 and 2 with a desirable point in week 9, however, during the intervention there was an undesirable trend in week 10 and 11. 
Figure 4

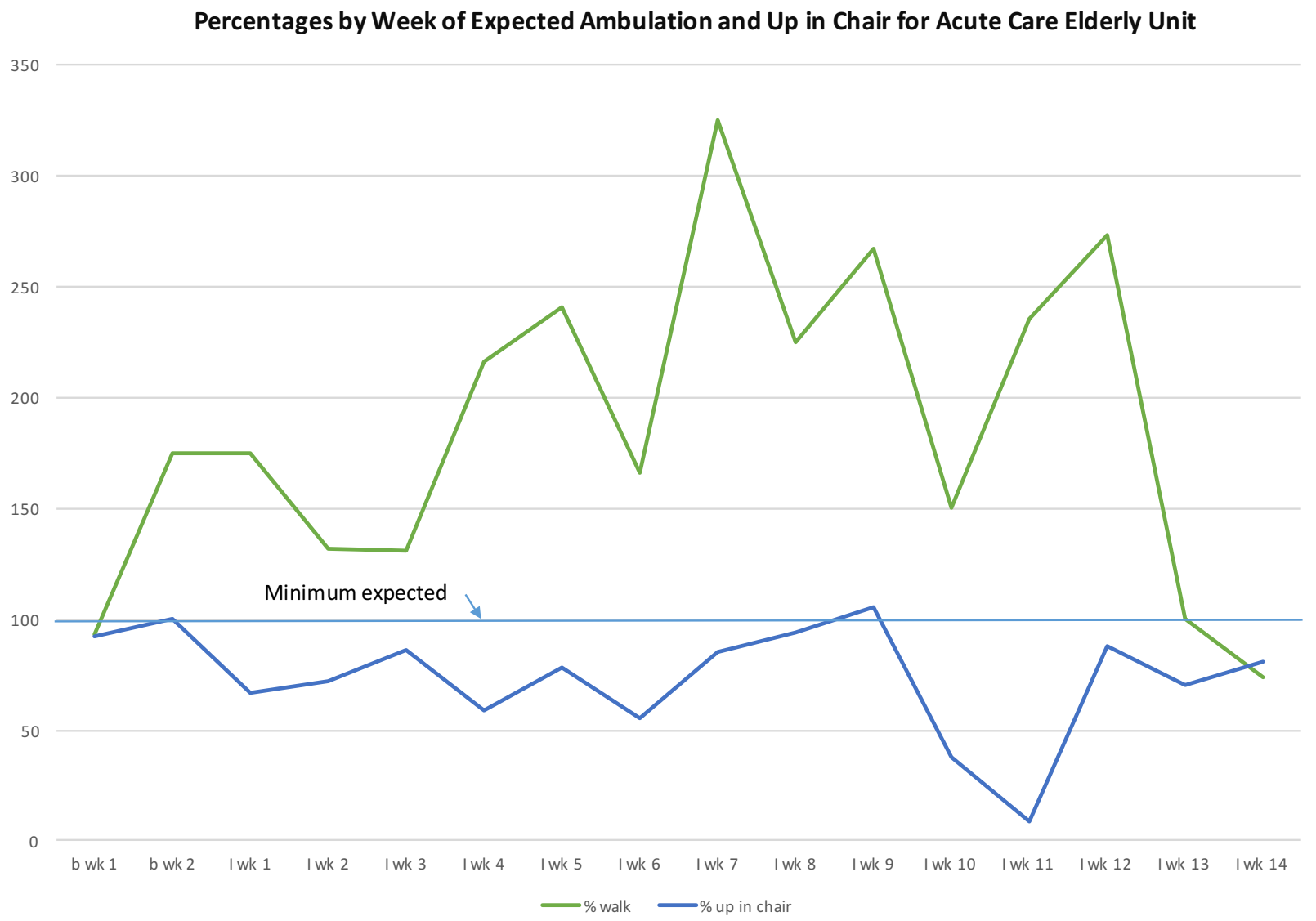

Figure 4 demonstrates that during the baseline period, the patient was in the chair more frequently, but was walking less. After the intervention, the patients were in the chair less, but were walking significanty more. One possible explanation was that the patients were so fatigued from walking that they opted to lay in bed and rest. 


\section{Figure 5}

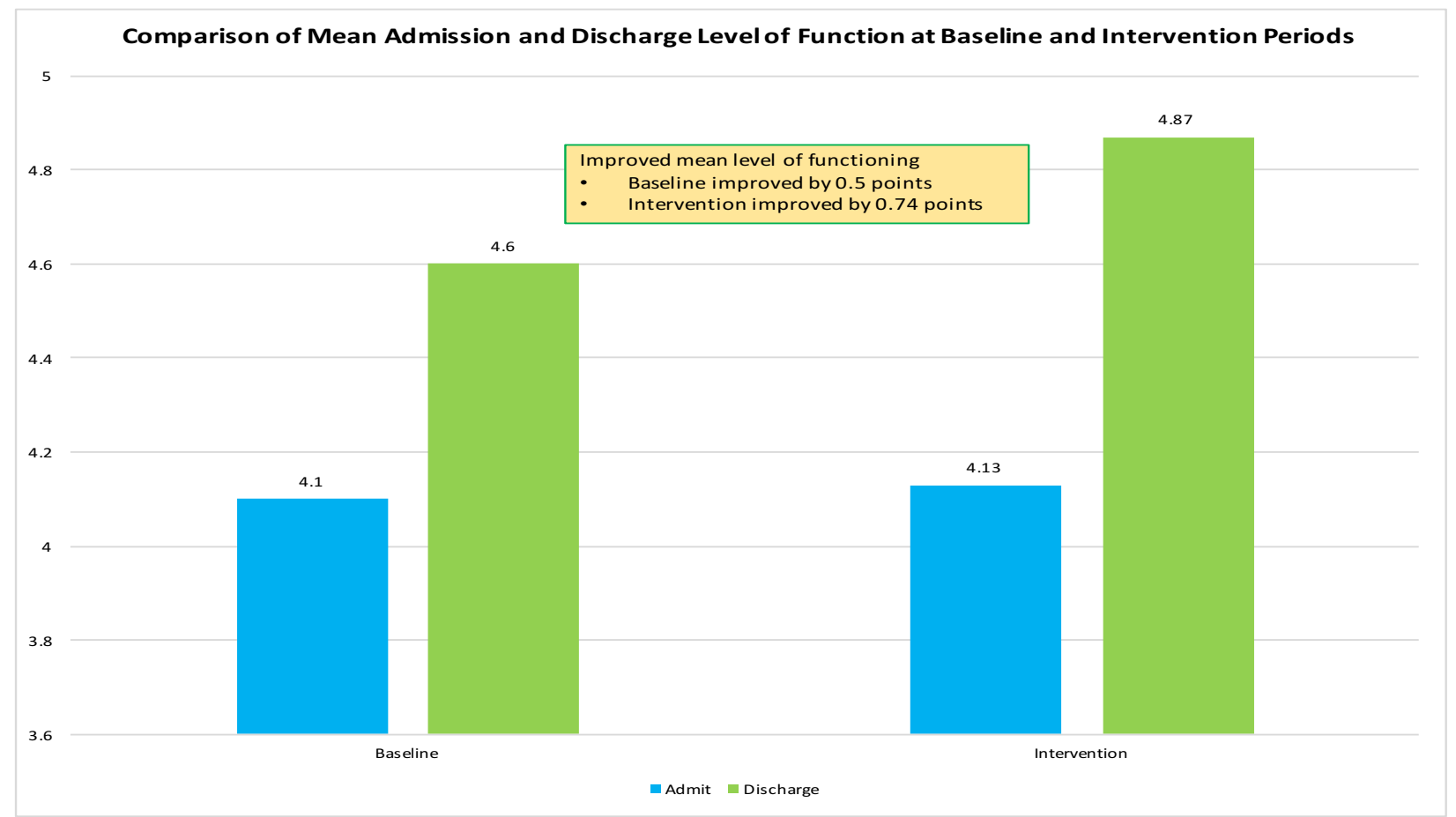

There was a mean improved level of function in the intervention by 0.74 points. Patients either maintained or improved their function at discharge. No patients in the intervention declined in function. Katz Activity of Daily Living score at baseline was 5.86, admission was 5.5, and discharge was 5.86. It is expected that patients may decline slightly when admitted to the hospital, but it was noted that the reported average was exactly the same as discharge function. This demonstrated that the intervention may have assisted the patients return to baseline ADL function. 


\section{Figure 6}

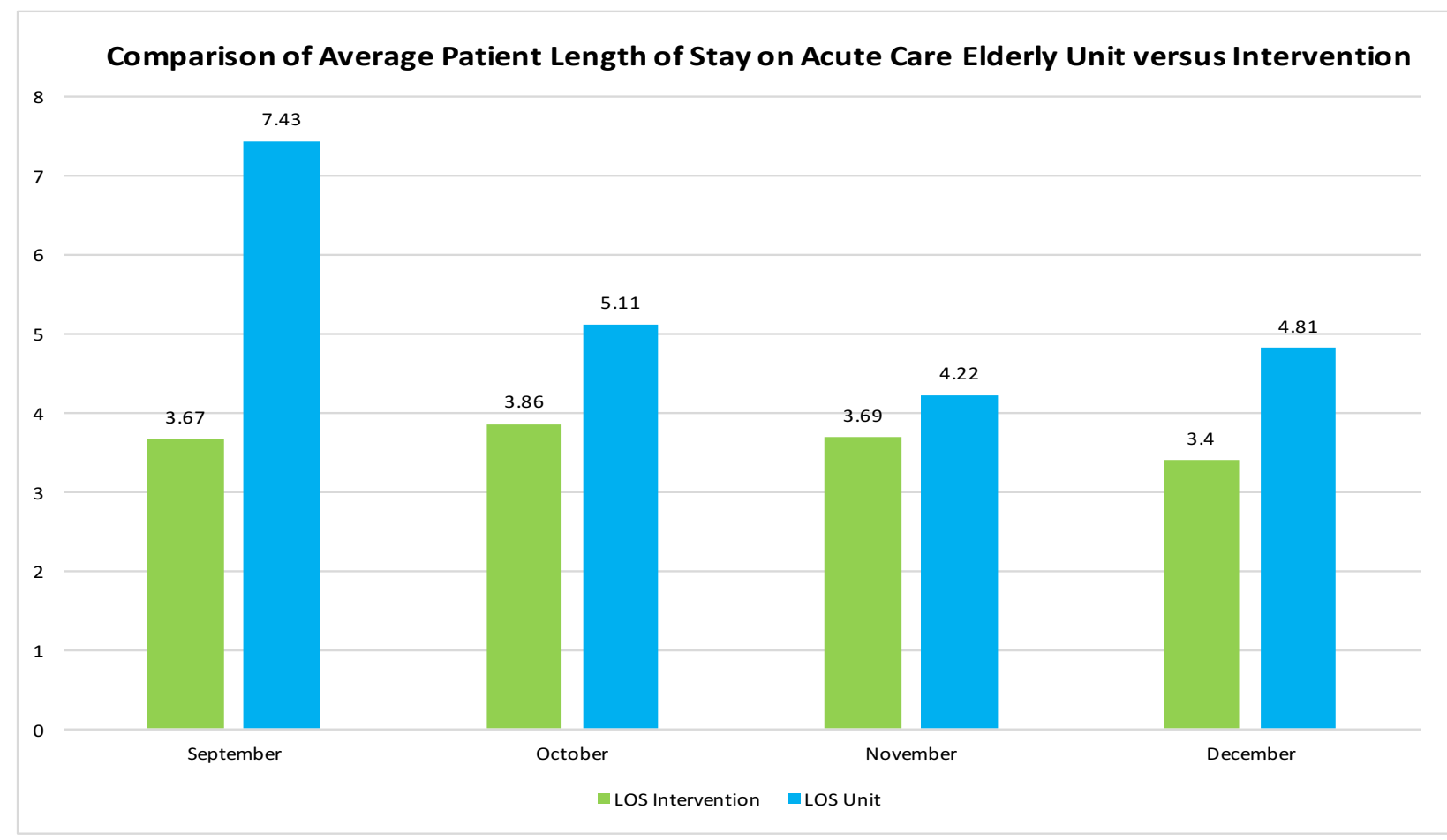

Overall length of stay in the patients in the intervention was decreased by about 1 day in comparison to the rest of the unit.

\section{Discussion}

This pilot project, like several written in the literature, were produced on small units with a small sample size and therefore may not be generalizable. However, in comparison with other studies mentioned, the patients in this study did not experience a fall or were not discharged to a SNF. Incidentally, three of the four patients that were transferred to other units were walking on the ACE unit and became bedbound after they were transferred. The fourth patient was walking with nursing staff on the ACE unit greater than 200 feet and when the patient was transferred to the other unit, the patient was walking only 50 feet and was being walked mostly with PT. Overall, it may be likely that length of stay was shorter because the patients were ambulatory on admission, whereas the rest of the unit included all patients, including those who were bedbound. 
Eleven days after project initiation, audit data showed some fall outs with float RNs. It was discussed that the patients identified for the study should be assigned to regular staff if there was a float RN. The charge RN was to assume the responsibilities or assist the float $\mathrm{RN}$ in education. Issues with Operating Room patient admissions were also addressed. Since the RN did not physically see them walk, it was assumed that they should be excluded from the study. It was then established that if the patient was walking before their surgery, that they would qualify for the study. There were several documentation omissions which may account for underestimating data. For example, some CNAs or RNs would select ambulation in the electronic medical record without documenting how many feet the patient walked. Throughout the study, it was difficult to have the patient in the chair three times a day. This may be explained by competing priorities, lack of documentation, or patient refusal. The Charge RNs took it upon themselves to add a section on their Charge RN report to ensure patient ambulation and education was completed. Overall, the RN staff did a wonderful job in correctly identifying patients for the study. However, of the 53 patients, two patients were identified two days after admission and two other patients were not identified at all. It was noted that these four patients still received the intervention because the staff appeared to adopt the intervention for all their patients.

There were several Katz tool sheets that were not returned to the folder. It was suggested by the DBT to change the color of the sheet in Month 2 on florescent paper so it would not be missed. This intervention did not make a difference. This could be for several reasons. Education was placed in the huddle book instead of one-on-one education by the GCNS or the DNP student, there were more patients in the last month of the study, the staff was losing traction towards the end, the DNP student was not present, or there was not sufficient time to notice a 
difference. The final and most likely reason that the Katz tool was not returned was that it was considered paper charting and therefore more cumbersome than charting in the electronic medical record.

Data analysis took one year to complete. The results were presented in early 2018 to the Assistant Department Administrator and Nurse Educator, the Department Administrator, GRNs, DBT, PT, Patient Education Committee, and Patient Member Committee, and to the new Mobility Committee.

\section{Cost-Benefit}

The overall project impact generated cost savings through a reduction in adverse events like falls and decreased LOS. Two weeks after discharge, hospitalized older adults are at risk for fall, which in some settings account for $15 \%$ of readmissions (Hastings et al., 2014). The cost of a fall is $\$ 35,000$, in addition to indirect costs of disability, reduced quality of life, and dependence on others (Centers for Disease Control and Prevention, 2015). No extra staff were needed and education was done during working hours. The following were free of charge: auditing, patient family handout creation, educational printing, and lamination. The cost for colored Katz tool fluorescent paper was fifteen dollars. Return on investment for preventing one fall is $233,300 \%$. The benefits may be underestimated since the indirect costs of disability, reduced quality of life, and dependence on others are not known and readily calculated.

\section{Conclusion}

\section{Current Practice}

In 2018, the study served to solidify the positive effects of a specialized ACE unit. The unit now has specific admission criteria which includes the patient being ambulatory prior to illness and must be 70 years or older. An educational handout of the effects of immobility did not 
exist at the organization and is now in the final stages of approval for hospital-wide use. The

ACE unit now has a check-off sheet on the unit for three types of mobilization or two

ambulations. The patients who are not ambulatory have range of motion exercises that are

counted as a mobilization instead of being placed in a chair. It is now the hospital-wide standard to have three mobilizations or two ambulations for each patient.

\section{Implications for Practice}

Maintaining mobility in the geriatric population benefits include reduction in cardiovascular disease, weight loss, reduced osteoporosis, enhanced sleeping, ability to perform ADLs, and improved mood and general health (Bruce \& Watt, 2006). Kaiser Permanente's mission is to provide high-quality, affordable healthcare services and to improve the health of our members and the communities we serve (Kaiser Permanente, 2016). The project assisted in improving the health of the members by maintaining mobility during hospitalization and discharging the patients back into the community.

A literature review of ACE units demonstrated overall better outcomes like decreased delirium, polypharmacy, LOS, readmission, total cost, discharge to nursing homes, improved ADLs, improved ambulation, and better patient and staff satisfaction compared to general medical surgical units (Ahmed \& Pearce, 2010). Maintaining or improving ADLs and ambulatory function during hospitalization is well documented in the literature and should be duplicated by other medical surgical units. Future studies would include a one-year follow-up for sustainability, larger sample size, or a comparison with another similar hospital unit that did not receive specialized education. 


\section{References}

Ahmed, N. N., \& Pearce, S. E. (2010). Acute care for the elderly: A literature review. Population Health Management, 13(4), 219-225. http://doi.org/10.1089/pop.2009.0058

Bruce, K., \& Watt, E. (2006). The effect if extra walking on the mobility, independence and exercise self-efficacy of elderly hospital in-patients: A pilot study. Contemporary Nurse, $22(1), 1-10$.

Centers for Disease Control and Prevention (2015). Costs of fall among older adults. Retrieved from http://www.cdc.gov/homeandrecreationalsafety/falls/fallcost.html

Creditor, M. C. (1993). Hazards of hospitalization of the elderly. Annals of Internal Medicine, $118(3), 219-223$.

Graf, C. (2006). Functional decline in hospitalized older adults. American Journal of Nursing, $106(1), 58-67$.

Hastings, S. N., Sloane, R., Morey, M. C., Pavon, J. M., \& Hoenig, H. (2014). Assisted early mobility for hospitalized older veterans: Preliminary data from the STRIDE program. Journal of the American Geriatrics Society, 62(11), 2180-2184.

http://doi.org/10.1111/jgs.13095

Kaiser Permanente (2016). About Kaiser Permanente. https://share.kaiserpermanente.org/about$\underline{\text { kaiser-permanente/ }}$

Katz, S., Downs, T. D., Cash, H. R., \& Grotz, R. C. (1970). Progress in Development of the Index of ADL. The Gerontologist, 10(1), 20-30.

Kleinpell, R.M., Fletcher, K., \& Jennings, B.M. (2008). Reducing functional decline in hospitalized elderly. In R.G. Hughes (Ed.), Patient safety and quality: An evidence-based handbook for nurses. Rockville, MD: Agency for Healthcare Research and Quality. 
Retrieved from

http://www.ncbi.nlm.nih.gov/books/NBK2629/pdf/Bookshelf_NBK2629.pdf

Kosse, N. M., Dutmer, A. L., Dasenbrock, L., Bauer, J. R. M., \& Lamoth, C. J. (2013).

Effectiveness and feasibility of early physical rehabilitation programs for geriatric hospitalized patients: a systematic review. BMC Geriatrics, 13(1), 1-16.

http://doi.org/10.1186/1471-2318-13-107

Krall, E., Close, J., Parker, J., Sudak, M., Lampert, S., \& Colonnelli, K. (2012). Innovation pilot study: Acute care for elderly (ACE) Unit_ promoting patient-centric care. Health Environments Research Design Journal, 5(3), 90-98.

Olson, E. V., Johnson, B. J., \& Thompson, L. F. (1990). The hazards of immobility. The American Journal of Nursing, 90(3), 43-48.

Padula, C. A., Hughes, C., \& Baumhover, L. (2009). Impact of a nurse-driven mobility protocol on functional decline in hospitalized older adults. Journal of Nursing Care Quality, $24(4), 325-331$.

Sare, M.V. \& Ogilvie, L. (2010). Strategic planning for nurses. Sudbury, MA: Jones \& Bartlett Learning.

Schaffer, M., Sandau, K., \& Diedrick, L. (2012). Evidenced-based practice models for organizational change: Overview and practical applications. Journal of Advanced Nursing, 69(5), 1197-1209. doi:10.1111/j.1365-2648.2012.06122.x

Titler, M. (2010). Iowa model of evidence-based practice. In J. Rycroft-Malone \& T. Bucknall (Eds.), Models and frameworks for implementing evidence-based practice: Linking evidence to action (pp.137-146). Malden, MA: Wiley-Blackwell. 
Valiani, V., Gao, S., Chen, Z., Swami, S., Harle, C. A., Lipori, G., .. Manini, T.M. (2016). Inhospital mobility variations across primary diagnoses among older adults. Journal of the American Medical Directors Association, 17(5), 465.e1-465.e8.

http://doi.org/10.1016/j.jamda.2016.02.003

Wallace, M., \& Shelkey, M. (2008). Katz index of independence in activities of daily living (ADL), American Journal of Nursing, 108(4), 64-71.

Wood, W., Tschannen, D., Trotsky, A., Grunawalt, J., Adams, D., Chang, R., ... DiccionMacDonald, S.(2014). A mobility program for an inpatient acute care medical unit, American Journal of Nursing, 114(10), 34-40.

http://doi.org/10.1097/01.NAJ.0000454850.14395.eb 